\title{
Ovarian Serous Cystadenofibroma- A Rare Tumour
}

\author{
Kiran Pande ${ }^{1}$, Unmesh Santpur², Tajinder Kaur ${ }^{3}$, Angela Yadav ${ }^{4}$ \\ ${ }^{1}$ Department of Obstetrics and Gynaecology, Maharishi Markandeshwar Institute of Medical Sciences and Research, \\ Mullana, Haryana, India. ${ }^{2}$ Department of Obstetrics and Gynaecology, Maharishi Markandeshwar Institute of Medical \\ Sciences and Research, Mullana, Haryana, India. ${ }^{3}$ Department of Obstetrics and Gynaecology, Maharishi \\ Markandeshwar Institute of Medical Sciences and Research, Mullana, Haryana, India. ${ }^{4}$ Department of Obstetrics and \\ Gynaecology, Maharishi Markandeshwar Institute of Medical Sciences and Research, Mullana, Haryana, India.
}

\section{INTRODUCTION}

Ovarian cystadenofibroma is a relatively rare benign ovarian tumour that contains both epithelial and fibrous stromal components. It is usually seen during the fourth and fifth decades. This tumour has features of malignancy in ultrasound and may also macroscopically appear as malignant during surgery. Since this tumour has a rare malignant potential, it is well-advised to be aware of the possibility of a cystadenofibroma before selecting an aggressive surgical approach in young patients. Ovarian cystadenofibroma is a relatively rare benign tumour that is seen in women aged 15-65 years.[1] The routine imaging features of this tumour may mimic a malignant neoplasm, but the presence of the fibrous component often gives a specific/characteristic MRI appearance that may help differentiate it from malignant ovarian tumours. ${ }^{[2-5]}$

\section{PRESENTATION OF CASE}

A 31-year-old female, para 2 live 2 with normal delivery 7 and 2 years back, presented in OPD with complaint of pain abdomen in right iliac fossa since 3 months, pain is increasing in intensity since last 6-7 days and gets relieved with medication and is associated with vomiting. Patient is also complaining of amenorrhea since 3 months. Her previous cycles were regular and associated with dysmenorrhoea. The urine pregnancy test was negative. On examination, patient was calm, conscious and well oriented to time, place and person. BP 140/90, PR 96/min regular and volume. Temp 98.6, saturation $99 \%$ on room air. Respiratory and cardiovascular systems were normal on examination. On per abdomen examination - soft, non-tender, obesity ++, cystic mass of $15 \times 10 \mathrm{~cm}$ size felt in right iliac fossa, mass was mobile and non-tender and of pelvic origin. On per speculum examination, vagina healthy and cervix hypertrophied and circumoral erosion + . On per vaginum examination normal uterus with a soft, mobile right adnexal mass was felt, cervical motion was not transmitted to the mass. On PR rectal mucosa was free.

Routine investigations were done $\mathrm{Hb} 11.4 \mathrm{~g} / \mathrm{dl}$, liver and renal function tests were normal and viral markers non-reactive. ESR was 20, TSH 2.6 and CA-125 was 10.60. A pelvic USG showed a large cystic lesion measuring $11.0 \mathrm{~cm} \times 9.6 \mathrm{~cm}$ with $\mathrm{few}$ internal echoes with no internal vascularity in the right adnexa, while the right ovary could not be separately identified, suggesting a possible ovarian origin. CT Scan of pelvis showed a well-defined complex cystic mass of size 12 × 12 ×8.5 cm arising from the right adnexa (right ovary was not separately identified) displacing adjacent bowel loops with preserved fat planes. Uterus was normal in size and was separate from the lesion. Pelvic vessels appeared normal and no pre or para aortic lymphadenopathy visualized. Pelvic fat planes were well maintained and appeared normal.
Corresponding Author: Dr. Angela Yadav,

Room No. 107, MMIMSR, MMU,

Mullana, Ambala,

Haryana, India.

E-mail: angelayadav93@gmail.com

DOI: $10.14260 / j e m d s / 2020 / 176$

Financial or Other Competing Interests: None.

How to Cite This Article:

Pande K, Santpur U, Kaur T, et al. Ovarian serous cystadenofibroma- a rare tumour. J. Evolution Med. Dent. Sci. 2020;9(10):814816, DOI: 10.14260/jemds/2020/176

Submission 03-01-2020,

Peer Review 11-02-2020,

Acceptance 19-02-2020,

Published 09-03-2020.

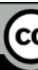




\section{DIFFERENTIAL DIAGNOSIS}

The history and examination of the patient is suggestive of a mass arising from the pelvis in the right iliac fossa which is separate from the uterus. At this stage our differential diagnosis includes benign or malignant ovarian tumours, most likely endometrioma, ovarian epithelial lesion, tubo ovarian abscess, parovarian cyst, mesenteric cyst and bladder tumour, after getting all the investigations done we concluded that the origin of the cyst is from the right ovary and but the nature of the tumour could not be made out. CT findings suggested towards the malignancy whereas the CA 125 levels were favouring the benign nature of the cyst.

\section{DISCUSSION OF MANAGEMENT}

An exploratory laparotomy was performed to confirm the diagnosis and to remove the cyst. Intraoperatively a cystic mass was visible of $11 \times 11 \times 5 \mathrm{~cm}$ arising from the right ovary separate from the uterus (figure 1). The right ovarian cyst was then dissected and sent for frozen section to determine the course of our surgery. Report of the frozen section of the right ovarian cyst was negative for malignancy and hence conservative approach was taken in the surgery. Right ovarian cystectomy was done and healthy ovary was left behind.
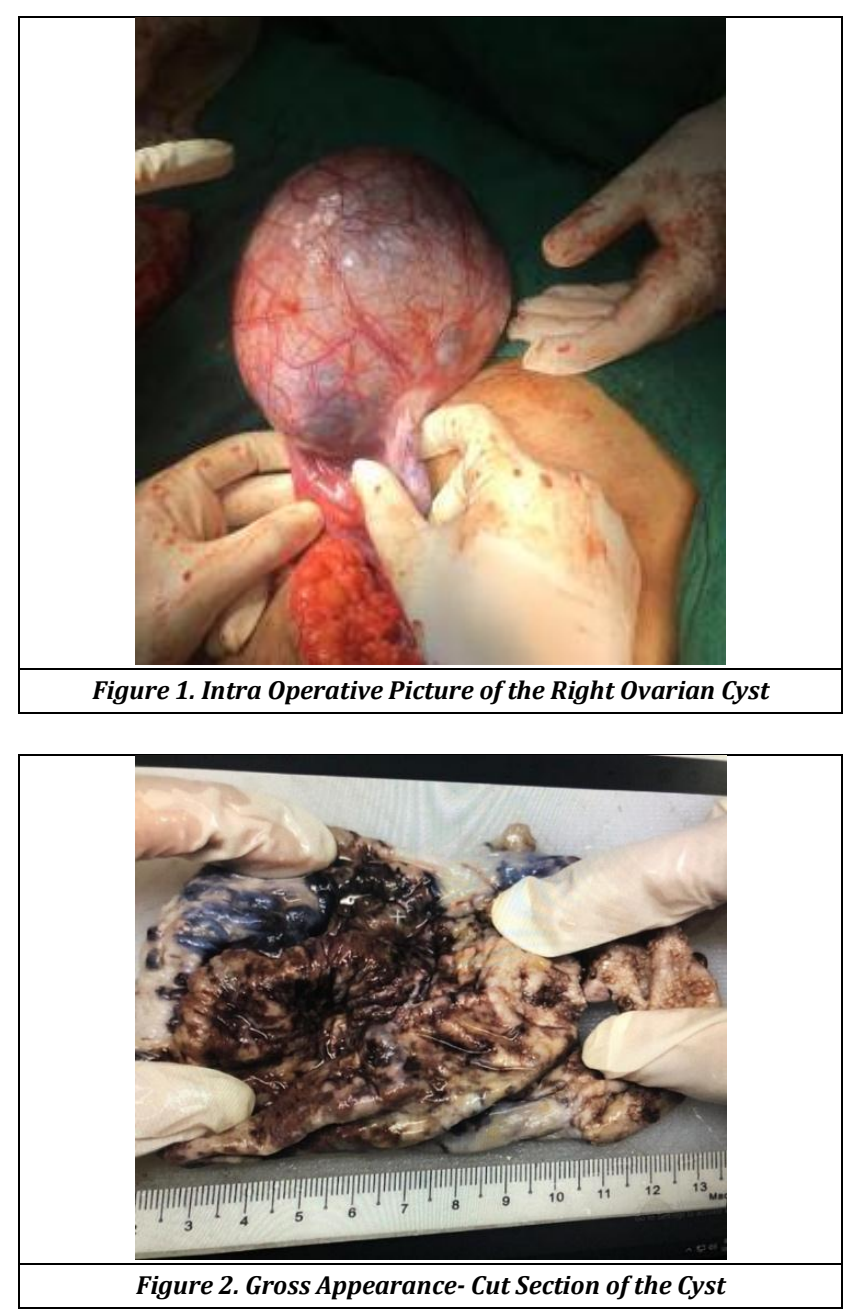

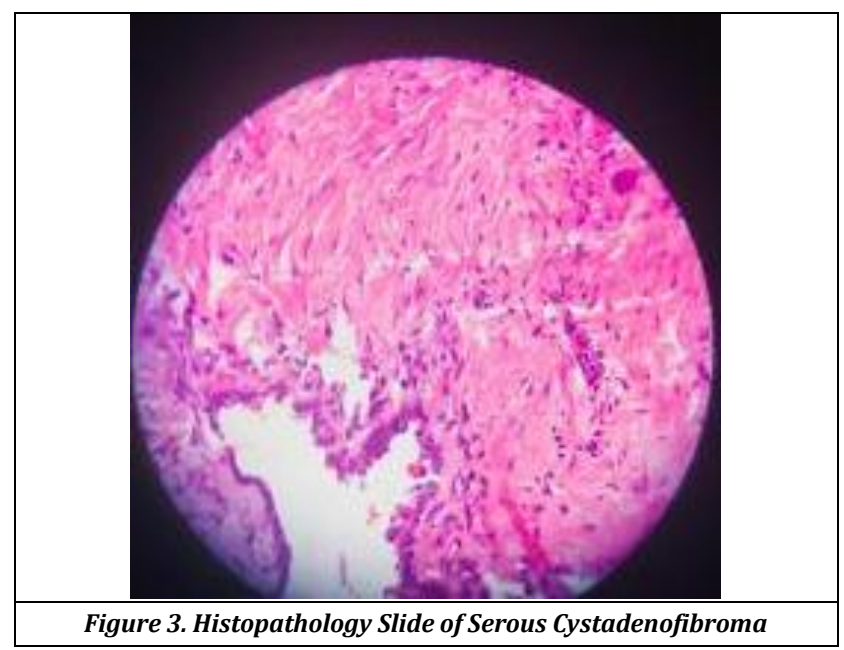

\section{PATHOLOGICAL DISCUSSION}

The frozen section of the right ovarian mass demonstrated a capsulated, soft tissue mass measuring $15 \times 12$ × $7 \mathrm{cms}$. on cut section, chocolate brown fluid extruded out (figure 2). Uniloculated cyst with multiple small cysts certified. The sample was negative for malignancy. Histopathology findings showed- a cyst marked denudation of lining epithelium with focal areas showing broad papillae with marked stromal fibrosis. The wall of the cyst was partly formed by fibroconnective tissue and partly by ovarian stroma. There was no evidence of stratification/atypia/invasion. Diagnosis was confirmed on histopathology report as serous cystadenofibroma of the right ovary (figure 3).

\section{DISCUSSION}

Ovarian cystadenofibroma is an uncommon benign neoplasm containing epithelial and fibrous stromal components. These tumours account for $1.7 \%$ of all benign ovarian tumours.[2] These tumours can be predominantly cystic, complex cystic with variable amounts of solid components or predominantly solid.[2,3] The presence of solid components and irregular thick septae, often leads to their misdiagnosis as malignant tumours on imaging. Even at the time of surgery, the gross appearance of a cystadenofibroma may resemble a malignant tumour. A frozen section diagnosis may be helpful in these cases as the correct diagnosis of cystadenofibroma in the operating room might save the patient from unnecessary extensive surgery. ${ }^{[2]}$ Cystadenofibroma may occur in reproductive age group and an accurate preoperative diagnosis may help in avoiding extensive surgical procedure.[4]

On sonography, it may show a unilocular cyst or a multiseptated cystic mass, with solid nodules. It may also show papillary projections. The solid components may show internal vascularity.[6] This heterogenous appearance can lead to a misdiagnosis of malignant ovarian neoplasm. Hence, Sonography can be used as a very good screening tool. However, MRI is better modality for proper characterization of the tumour. A computed tomography (CT) scan also has limited value in evaluating this tumour. In a study by Cho et al., all 16 cases, presented as complex solid-cystic masses which were preoperatively misdiagnosed as malignant ovarian 
neoplasms on CT scan or MRI and were proven to be ovarian cystadenofibroma on histopathology.[2]

Contrast enhanced MRI has been proved as the investigation of choice for characterizing complex ovarian solid-cystic masses. Outwater et al.[5] first described the MRI feature of cystadenofibroma, appearing hypointense on $\mathrm{T} 2 \mathrm{~W}$ sequences (relative to the skeletal muscle) representing the solid fibrous component. Multiple tiny hyperintense -T2-signal intensity cysts are also seen to be associated with it giving it a sponge-like appearance on T2 images [4]. Multicystic foci with thickened septae, demonstrating low-T2-signal intensity corresponding to its fibrous nature.[3]

Fibroma, fibrothecoma and Brenner tumour, which are all benign tumours are other tumours having similar appearance on MRI because of the fibrous component.

\section{REFERENCES}

[1] Czernobilsky B, Borenstein R, Lancet M. Cystadenofibroma of the ovary. A clinicopathologic study of 34 cases and comparison with serous cystadenoma. Cancer 1974;34(6):1971-81.
[2] Cho SM, Byun JY, Rha SE, et al. CT and MRI findings of cystadenofibromas of the ovary. Eur Radiol 2004;14(5):798-804

[3] Jung DC, Kim SH, Kim SH. MR imaging findings of ovarian cystadenofibroma and cystadenocarcinofibroma: clues for differential diagnosis. Korean J Radiol 2006;7(3):199204.

[4] Takeuchi M, Matsuzaki K, Kusaka M, et al. Ovarian cystadenofibromas: characteristic magnetic resonance findings with pathologic correlation. J Comput Assist Tomogr 2003;27(6):871-3.

[5] Outwater EK, Siegelman ES, Talerman A, et al. Ovarian fibromas and cystadenofibromas: MRI features of the fibrous component. J Magn Reson Imaging 1997;7(3):465-71.

[6] Alcázar JL, Errasti T, Mínguez JA, et al. Sonographic features of ovarian cystadenofibromas: spectrum of findings. J Ultrasound Med 2001;20(8):915-9. 